Erteilung und Widerruf von

\title{
Abrechnungsgenehmigungen in der Radiologie nach § 135 Abs. 2 SGB V
}

\section{Qualitätssicherung in der vertragsärztlichen Versorgung}

Im 9. Abschnitt des 4. Kapitels des 5. Buches Sozialgesetzbuch (SGB V) hat der Gesetzgeber die „Sicherung der Qualität der Leistungserbringung “ geregelt. Dieser Abschnitt umfasst neben einer Bewertung von Untersuchungs- und Behandlungsmethoden in der ambulanten und stationären Versorgung durch den Gemeinsamen Bundesausschuss (G-BA) nach den $\S \S$ 135 Abs. 1, 137c, die Verpflichtung der Leistungserbringer zur Qualitätssicherung nach $\S 135$ a und einer korrelierenden Förderungspflicht durch die Kassenärztlichen Vereinigungen ( $\S 136$ ) sowie die Bewertung und Weiterentwicklung der Maßnahmen zur Qualitätssicherung über und durch den G-BA ( $\$ 137 a$ ff.), Regelungen über strukturierte Behandlungsprogramme (§ 137f), der Qualitätssicherung in der Heil- und Hilfsmittelversorgung ( $\S \S 138,139)$ und die Bestimmungen über das Institut für Qualität und Wirtschaftlichkeit im Gesundheitswesen (IQWiG).

Die Maßnahmen der Qualitätssicherung leiten sich in der vertragsärztlichen Versorgung aus $\S 135$ Abs. 2 SGB V ab. Danach gilt, dass die Partner der Bundesmantelverträge ( $§ \S 82,85$ SGB V), also die Kassenärztliche Bundesvereinigung und der Spitzenverband Bund der Krankenkassen für ärztliche und zahnärztliche Leistungen, welche wegen der Anforderungen an ihre Ausführung oder wegen der Neuheit des Verfahrens

- a besonderer Kenntnisse und Erfahrungen (Fachkundenachweis),

- b einer besonderen Praxisausstattung,

- c Anforderungen an die Versorgungsqualität

bedürfen, einheitliche Voraussetzungen für die Ausführung und Abrechnung dieser Leistungen vereinbaren können. Sind bereits für die notwendigen Kenntnisse und Erfahrungen, welche als Qualifikation vorausgesetzt werden müssen, in landesrechtlichen Regelungen zur ärztlichen Berufsausübung, insbesondere solchen des
Facharztrechts, bundesweit inhaltsgleiche und hinsichtlich der Qualitätsvoraussetzungen, die durch den Bundesmantelvertrag gefordert werden, gleichwertige Qualifikationen eingeführt worden, sind diese notwendige und ausreichende Voraussetzung. Hintergrund dieser Regelung ist, dass der Gesetzgeber von einem einheitlichen Berufsbild des „Arztes“ ausgehen will und es sich bei der vertragsärztlichen Tätigkeit nur um eine bestimmte Ausprägung des ärztlichen Berufs handelt (vgl. BVerfGE 11, 30). Der Fachkundenachweis als Nachweis besonderer Kenntnisse und Erfahrungen innerhalb eines Fachgebiets findet sich nicht nur in $\S 135$ Abs. 2 SGB V, sondern insbesondere in den Weiterbildungsordnungen der einzelnen Ärztekammern. Damit gehen die landesrechtlichen Berufsregelungen den Qualitätsanforderungen vor, sofern die Regelungen über alle Ärztekammern hinweg inhaltsgleich und hinsichtlich der Qualitätsvoraussetzungen eine gleichwertige Qualifikation erfordern und der Gesetzgeber nicht gegenüber dem Weiterbildungsrecht abweichende oder weitergehende Qualitätsanforderungen verlangt.

Wird die Erbringung ärztlicher Leistungen erstmalig von einer Qualifikation abhängig gemacht, so können die Vertragspartner nach $\S 135$ Abs. 2 SGBV für Ärzte, welche entsprechende Qualifikationen nicht während einer Weiterbildung erworben haben, übergangsweise Qualifikationen einführen, welche dem Kenntnis- und Erfahrungsstand der facharztrechtlichen Regelungen entsprechen müssen.

Für Leistungen, die zum Kernbereich gehören, sieht $\S 135$ Abs. 2 SGB V eine besonders für vertragsärztlich tätige Radiologen bedeutsame Regelung vor. Demnach können die Vertragspartner abweichend von den vorstehenden Regelungen zur Sicherung der Qualität und der Wirtschaftlichkeit der Leistungserbringung Regelungen treffen, nach denen die Erbringung bestimmter medizinisch-technischer Leistungen den Fachärzten vorbe- halten ist, für die diese Leistungen zum Kern ihres Fachgebiets gehören. Dies hat insbesondere Bedeutung für die Erbringung von Leistungen der Kernspintomografie, die nach wie vor in der vertragsärztlichen Versorgung, aufgrund der Qualitätssicherungsvereinbarungen, ausschließlich durch Fachärzte für Radiologie erbracht werden dürfen, auch wenn Orthopäden und Kardiologen nach Erhalt der Zusatzweiterbildung „fachgebundene Magnetresonanztomografie“ privatärztlich zur Erbringung dieser Leistungen berechtigt sind.

\section{Qualitätssicherung in der Radiologie \\ $\nabla$}

Besonders im Fachgebiet der Radiologie bedarf ein Vertragsarzt neben seiner $\mathrm{Zu}$ lassung zur vertragsärztlichen Versorgung einer Reihe von Genehmigungen, um bestimmte vertragsärztliche Leistungen erbringen und abrechnen zu dürfen. So bestehen neben den Richtlinien der Kassenärztlichen Bundesvereinigung für Verfahren zur Qualitätssicherung nach § 75 Abs. 7 SGB V (Qualitätssicherungsvereinbarungen der KBV und des Spitzenverbands Bund) gemäß § 135 Abs. 2 SGB V

- die Vereinbarung von Qualitätssicherungsmaßnahmen zur MR-Angiografie (MR-Angiografievereinbarung),

- die Vereinbarung von Qualitätssicherungsmaßnahmen zur Ultraschalldiagnostik (Ultraschall-Vereinbarung),

- die Vereinbarung von Qualifikationsvoraussetzungen zur Durchführung von Untersuchungen in der diagnostischen Radiologie und Nuklearmedizin und von Strahlentherapie (Vereinbarung zur Strahlendiagnostik und -therapie), 
- oder die Qualitätssicherungsmaßnahme zur kurativen Mammografie

(Mammografie-Vereinbarung),

- Kernspintomografie-Vereinbarung.

Trotz grundsätzlicher Ähnlichkeiten der Qualitätsvereinbarungen unterscheiden sie sich nicht nur in den fachlichen Voraussetzungen, sondern in den Genehmigungs-, Widerrufs- und Wiedererteilungsverfahren.

\section{Genehmigungserteilung \\ $\nabla$}

Die Ausführung und Abrechnung von radiologischen Leistungen in der vertragsärztlichen Versorgung durch vertragsärztlich tätige Radiologen ist grundsätzlich erst nach Erteilung der Genehmigung durch die Kassenärztliche Vereinigung zulässig. Die Kassenärztliche Vereinigung hat die Genehmigung zu erteilen, wenn der Arzt die fachlichen, apparativen und organisatorischen Voraussetzungen nach den Bestimmungen der betreffenden Vereinbarung im Einzelnen erfüllt. Das Genehmigungsverfahren richtet sich nicht nur nach der betreffenden Qualitätssicherungsvereinbarung, sondern ergänzend nach den Richtlinien der Kassenärztlichen Bundesvereinigung für Verfahren zur Qualitätssicherung gemäß § 75 Abs. 7 SGB V. Nach der erteilten Genehmigung ist der Radiologe berechtigt, die genehmigten Leistungen für gesetzlich krankenversicherte Patienten gegenüber der Kassenärztlichen Vereinigung zu erbringen und abzurechnen.

\section{Widerruf der Genehmigung}

Immer wieder widerrufen die Kassenärztlichen Vereinigungen Genehmigungen zur Erbringung von Kernspintomografien, Röntgenleistungen oder Sonografien gegenüber Vertragsärzten. Als Beispiel soll ein Fall dienen, über den das Landessozialgericht Hamburg am 07.06.2012 (Az.: L 1 KA 65/09) zu entscheiden hatte. Das Landessozialgericht bestätigte in seinem Urteil den Widerruf einer Abrechnungsgenehmigung für sonografische Leistungen eines Urologen durch die zuständige Kassenärztliche Vereinigung. Die Kassenärztliche Vereinigung hatte den Urologen Jahre zuvor aufgefordert, zur Qualitätskontrolle im Quartal IV/2004 seine Ultraschalldokumentationen und Befunde für 9 bestimmte Patienten zu übersenden. Die Dokumentationen betra- fen 8 rektale und 1 abdominale Untersuchung.

Die Sonografie-Kommission kam in ihrer Sitzung, in der die eingereichten Ultraschall-Dokumentationen zu bewerten waren, zu keinem abschließenden Ergebnis. Die übersandten Aufnahmen seien überwiegend von nicht ausreichender Qualität. Der Urologe wurde aufgefordert, zur weiteren Beurteilung 5 neu erstellte Dokumentationen seiner Wahl einzureichen. Die Sonografie-Kommission kam im Folgejahr zum Ergebnis, dass die überlassenen rektalen Ultraschalldokumentationen beanstandungsfrei seien. Allerdings wies die Nierensonografie, für die nur eine Dokumentation vorgelegt worden war, erhebliche Mängel bezüglich der Nachvollziehbarkeit der Echomuster und der Organgrenzen beider Nieren auf. Der Urologe wurde sodann gebeten, zur weiteren Beurteilung 5 neu erstellte Dokumentationen eigener Wahl, auf denen beide Nieren und Harnblase abgebildet sein sollten, einzureichen. Dem kam der Urologe nicht nach, sondern teilte mit, dass er die Prüfung als abgeschlossen und bestanden betrachte, da zunächst ausschließlich transrektale Ultraschallbilder angefordert worden seien, die nunmehr ohne Beanstandung seien. Einer Erinnerung an die Übersendung der weiteren Aufnahmen kam der Urologe nicht nach. In der Folgezeit widerrief die Kassenärztliche Vereinigung die Sonografie-Genehmigung ab Zugang des Bescheids und verband dies mit der Anordnung der sofortigen Vollziehung, sodass der Urologe ab dem Zeitpunkt des Zugangs des Widerrufs keine sonografischen Leistungen mehr gegenüber gesetzlich versicherten Patienten erbringen und abrechnen durfte.

Es stellt sich jedoch prinzipiell die Frage, unter welchen Voraussetzungen ein Widerruf der Genehmigung zulässig ist. Ausgangspunkt dieser Frage ist der Genehmigungsbescheid, der heute durchgängig einen sogenannten Widerrufsvorbehalt oder eine Auflage enthält. Die Auflage ist inhaltlich, z.B. im Falle einer Genehmigung nach der Mammografie-Vereinbarung dahingehend bestimmt, dass der Vertragsarzt alle Anforderungen der Röntgenverordnung, insbesondere $\S 18 \mathrm{a}$ RöV, erfüllen muss. Der Widerrufsvorbehalt im Genehmigungsbescheid lautet etwa bei der Kassenärztlichen Vereinigung Schleswig-Holstein:

Die Genehmigung ist zu widerrufen, wenn
- die genannten Auflagen nicht erfüllt werden,

- die Qualität den geforderten Anforderungen nicht entspricht,

- die apparative Ausstattung nicht mehr den jeweils gültigen Richtlinien entspricht oder

- die Genehmigung unter unrichtigen Angaben erteilt wurde.

Nicht jede Kassenärztliche Vereinigung verfasst den Widerrufsvorbehalt in dieser Klarheit.

Im Falle der Angabe von unrichtigen Angaben bedürfte es eines vorbehaltenen Widerrufs nicht, weil aufgrund der gesetzlichen Bestimmungen im 10. Buch Sozialgesetzbuch (SGB X) eine Rechtsgrundlage besteht, den Genehmigungsbescheid zurückzunehmen. Ein Genehmigungsbescheid durch die Kassenärztliche Vereinigung darf daher nur nach Ausübung des pflichtgemäßen Ermessens mit einem Widerrufsvorbehalt nach $\S 32$ Abs. 2 SGB $X$ versehen werden. $\S 32$ des SGB X lautet in Auszügen wie folgt:

„§ 32 Nebenbestimmungen zum Verwaltungsakt

(1) Ein Verwaltungsakt, auf den ein Anspruch besteht, darf mit einer Nebenbestimmung nur versehen werden, wenn sie durch Rechtsvorschrift zugelassen ist oder wenn sie sicherstellen soll, dass die gesetzlichen Voraussetzungen des Verwaltungsaktes erfüllt werden.

(2) Unbeschadet des Absatzes 1 darf ein Verwaltungsakt nach pflichtgemäßem Ermessen erlassen werden mit [...]

3. einem Vorbehalt des Widerrufs oder verbunden werden mit

4. einer Bestimmung, durch die dem Begünstigten ein Tun, Dulden oder Unterlassen vorgeschrieben wird (Auflage), $[\ldots]^{*}$

$\S 32$ Abs. 1 Alt. 2 SGB X bestimmt für den Fall des sog. gebundenen Ermessens, dass auch ein Verwaltungsakt mit einer Nebenbestimmung - hier also einer Auflage - versehen werden darf, wenn diese sicherstellen soll, dass die gesetzlichen Voraussetzungen des Verwaltungsakts erfüllt werden. In dem Beispielsfall sollte nach der Vorstellung des Landessozialgerichts Hamburg der Vorbehalt des Widerrufs sicherstellen, dass auf Dauer die ge- 
setzlichen Voraussetzungen z.B. im Hinblick auf die Röntgenverordnung für die erteilte Genehmigung vorliegen.

Soweit ein Widerruf im Genehmigungsbescheid vorbehalten worden ist und der Genehmigungsbescheid mit einer Auflage versehen werden durfte, bedarf es dennoch für die Ausübung des Widerrufs einer weiteren gesetzlichen Grundlage. Diese findet sich generell für einen begünstigenden rechtmäßigen Verwaltungsakt, der eine Abrechnungsgenehmigung darstellt, in $\S 47$ SGB X.

\section{$\S 47$ Abs. 1 SGB X lautet:}

„§ 47 Widerruf eines rechtmäßigen begünstigenden Verwaltungsaktes

(1) Ein rechtmäßiger begünstigender Verwaltungsakt darf, auch nachdem er unanfechtbar geworden ist, ganz oder teilweise mit Wirkung für die Zukunft nur widerrufen werden, soweit

1. der Widerruf durch Rechtsvorschrift zugelassen oder im Verwaltungsakt vorbehalten ist,

2. mit dem Verwaltungsakt eine Auflage verbunden ist und der Begünstigte diese nicht oder nicht innerhalb einer ihm gesetzten Frist erfüllt hat.“

Ob die Voraussetzungen des Widerrufs vorliegen oder nicht und ob die Auflage erfüllt wurde oder nicht, sind indes Tatbestandsfragen des konkreten Einzelfalls und bedürfen sodann einer individuellen Prüfung im Einzelfall.

\section{Aufschiebende Wirkung des Widerspruches}

Sofern die Kassenärztliche Vereinigung die Abrechnungsgenehmigung widerrufen hat, steht dem Radiologen die Möglichkeit des Widerspruchs und - wenn dieser fruchtlos bleibt - die Klage vor dem Sozialgericht gegen diese Entscheidung zu.

Dem Widerspruch und einer ggf. erhobenen Anfechtungsklage gegen den Widerruf der Genehmigung kommt, wie in den meisten Verwaltungsverfahren, aufschiebende Wirkung zu, sodass der Radiologe während des gesamten Widerspruchsverfahrens oder des Klageverfahrens die Leistungen weiterhin erbringen und abrech- nen darf. Die Kassenärztlichen Vereinigungen machen unter dem Aspekt des Patientenschutzes jedoch häufig von der Möglichkeit der Anordnung des Sofortvollzugs nach $\S$ 86a Abs. 2 Nr. 5 SGG Gebrauch. Eine solche Anordnung des Sofortvollzugs bedarf nach $\S 86 a$ Abs. $2 \mathrm{Nr} .5$ SGG einer besonderen Begründung, die über ein grundsätzlich anzunehmendes Interesse an dem Vollzug eines aus Sicht der Kassenärztlichen Vereinigung rechtmäßigen Verwaltungsaktes hinausgehen muss. An dieser Begründung fehlt es allerdings regelmäßig, wenn wie in dem Beispielsfall des Landessozialgerichts Hamburg nur eine einzige Nierensonografie zu beanstanden war. Wenn alle im Übrigen begutachteten Leistungen beanstandungsfrei waren, gebietet es die Abwägung zwischen dem Patientenschutz und dem Grundrecht des Vertragsarztes auf Berufsausübungsfreiheit aus Art. 12 Abs. 1 des Grundgesetzes (GG), dass der Radiologe nur bestimmte Leistungen, die sich anhand der Angabe von Gebührenordnungspositionen des EBM ohne Weiteres angeben lassen, während des Widerspruchs- und Klageverfahrens nicht erbringen darf. Eines Nachweises, dass aus dem seitens der jeweiligen Kommission festgestellten Mangel eine Gefahr für die Patienten folgt, wird von der Rechtsprechung regelmäßig angenommen, weil diese eine abstrakte Gefahr für die Patienten genügen lässt. Wenn zum Beispiel die Dokumentation nicht vollständig war und dadurch ein nachbehandelnder Arzt nicht sämtliche Informationen erhalten konnte, kommt es daher nach der Rechtsprechung nicht darauf an, ob der nachbehandelnde Arzt tatsächlich auf die nicht dokumentierte Information angewiesen war oder nicht.

In diesem Zusammenhang stellt sich für den Radiologen die wichtige Frage, ob er das Honorar, das er aufgrund einer aufschiebenden Wirkung (sog. Suspensiveffekt) während des Widerspruchsverfahrens und ggf. des anschließenden Klageverfahrens von der Kassenärztlichen Vereinigung erhalten hat, an diese zurückzahlen muss, wenn in letzter Instanz festgestellt wird, dass die Genehmigung zur Leistungserbringung und Abrechnung nicht vorlag, weil sie rechtmäßig widerrufen worden war. Aufgrund der aufschiebenden Wirkung bestand die jeweilige Genehmigung zur Erbringung und Abrechnung von vertragsärztlichen Leistungen prinzipiell fort. Leistungen, die während dieser Zeit erbracht wurden, können daher abgerechnet werden und werden nicht nachträglich als rechtswidrig bzw. rückwirkend ohne Genehmigung erbracht angesehen. Die Wirkung des Widerrufs der Genehmigung tritt in diesen Fällen erst dann ein, wenn dieser bestandskräftig wird; sei es durch Verzicht auf ein Rechtsmittel, aufgrund der Rücknahme eines Rechtsmittels oder durch eine rechtskräftige Entscheidung eines Sozialgerichts. Eine Grundlage für die Rückforderung während einer solchen rechtlichen Schwebephase erbrachter vertragsärztlicher Leistungen besteht für die Kassenärztliche Vereinigung nicht.

Mit seiner Entscheidung vom 23.06.2010 (Az.: B 6 KA 7/09 R) hat das Bundessozialgericht zwar bestehende Tendenzen der Kassenärztlichen Vereinigungen über sachlich-rechnerische Berichtigungen, Honorare zurückzufordern, bestätigt, wenn die zulassungsrechtlichen Voraussetzungen von Anstellungen, Berufsausübungsgemeinschaften oder Medizinischen Versorgungszentren nur formal vorlagen. In jenen Fällen bestanden die Bindungswirkung und der Vertrauensschutz, die aus den Zulassungsbescheiden aufgrund der Bestandskraft folgten, nur zwischen den Zulassungsgremien und den Leistungserbringern. Die Kassenärztliche Vereinigung soll in diesen Fällen nach Ansicht des Bundessozialgerichts nicht an die Entscheidung der Zulassungsgremien gebunden sein. Im Falle der hier relevanten Abrechnungsgenehmigung, die seitens der Kassenärztlichen Vereinigung erteilt wurde, besteht die Bindung nur zwischen der Kassenärztlichen Vereinigung und dem Vertragsarzt, sodass hier der Vertrauensschutz gerade gegenüber der Kassenärztlichen Vereinigung besteht und diese das Instrument der sachlichrechnerischen Berichtigung nicht verwenden kann.

\section{Wiedererteilung der Genehmigung $\nabla$}

Der Widerruf einer Abrechnungsgenehmigung ist mit einem gewissen Pragmatismus betrachtet, für den betroffenen Vertragsarzt selten von existenzbedrohender Bedeutung, wie dies etwa bei einer Zulassungsentziehung der Fall ist. Nach einem Widerruf der Genehmigung kann diese durch die Beantragung einer Wiedererteilung nach einem Zeitraum von 3 Monaten, im Falle eines Verstoßes gegen die Qualitätssicherungs-Richtlinie 
und z.B. nach 6 Monaten bei einem Verstoß gegen die MR-Angiografievereinbarung wiedererlangt werden.

Im Einzelfall dauert das Wiedererteilungsverfahren allerdings mehrere Monate, weil z.B. die QualitätssicherungsRichtlinie vorsieht, dass sich die Kassenärztliche Vereinigung vor der Wiedererteilung einer Genehmigung durch ein Kolloquium vor einer QualitätssicherungsKommission bzgl. der Erfüllung der Anforderungen seitens des Antragstellers vergewissern kann, wenn trotz der Erfüllung der formalen Voraussetzungen aufgrund der vorgelegten Zeugnisse und Bescheinigungen dennoch Zweifel an der fachlichen Befähigung bestehen. Die zuständigen Qualitätssicherungskommissionen tagen allerdings zum Teil nur alle 6 Monate, sodass der Zeitraum bis zu einer Wiedererteilung deutlich über der „Wartezeit“ liegt. In den Fällen, in denen die Kassenärztliche Vereinigung den Sofortvollzug angeordnet hat, sollte stets nach Ablauf der Wiedererteilungsfrist unverzüglich ein Antrag auf Wiedererteilung gestellt werden, um die genehmigungsbedürftigen Leistungen erneut erbringen und $a b$ rechnen zu dürfen.

Das Wiedererteilungsverfahren ist in den verschiedenen Qualitätssicherungsvereinbarungen unterschiedlich geregelt. Das Wiedererteilungsverfahren nach der MR-Angiografie-Vereinbarung richtet sich z.B. nicht nach dem (Erst-)Genehmigungsverfahren, sondern nach der fachlichen Befähigung, apparativen und organisatorischen Voraussetzungen, Befundung und Bildnachbearbeitung gemäß den $\S \S 3$ bis 5 der MR-Angiografie-Vereinbarung. Andererseits ist ein Kolloquium nach der MR-Angiografie-Vereinbarung im Falle einer Wiedererteilung - anders als in bestimmten Fällen der Erstgenehmigung nicht vorgesehen.

Im Falle des Widerrufs einer Sonografiegenehmigung nach $\S 11$ Abs. 6 Satz 2 der Ultraschall-Vereinbarung kann der Arzt frühestens nach Ablauf von 3 Monaten nach dem Widerruf der Genehmigung einen Antrag auf eine erneute Teilnahme an einem Kolloquium stellen. Diesem Antrag ist eine Bescheinigung über die zwischenzeitliche Teilnahme an geeigneten Fortbildungsmaßnahmen beizulegen. Ist die Teilnahme an dem Kolloquium erfolgreich, erteilt die Kassenärztliche Vereinigung die Genehmigung.

\section{Zusammenfassung und Ausblick}

$\nabla$

Das Verfahren auf Widerruf der Genehmigung ist in den verschiedenen Qualitätssicherungsvereinbarungen sehr unterschiedlich geregelt und nicht an das Verwaltungsverfahren des SGB X angelehnt. Das Verfahren ist daher aus rechtsstaatlicher Sicht zu kritisieren. Dennoch entscheiden die Sozialgerichte selten zugunsten des Vertragsarztes, dem die Genehmigung entzogen worden ist. Diese Entscheidungen beruhen häufig auf der Annahme einer drohenden Gefahr für die Patienten, die seitens der Sozialgerichte gelegentlich sehr unkritisch angenommen wird und dem Grundrecht des betroffenen Radiologen auf Berufsausübung gemäß Art. 12 Abs. 1 GG zuwiderlaufen. Rechtsmittelverfahren gegen den Widerruf einer Abrechnungsgenehmigung sind präzise zu führen und bedürfen neben einer Betrachtung der Formalien einer eingehenden Betrachtung der seitens der Kassenärztlichen Vereinigung erhobenen Vorwürfe. In der Regel ist die Einholung eines Sachverständigengutachtens erforderlich, um das Sozialgericht von der unzutreffenden Beurteilung durch die jeweilige Kommission zu überzeugen. Bevor daher ein langwieriger Rechtsstreit durch den Radiologen geführt wird, sollte geprüft werden, ob durch die Praxisorganisation Möglichkeiten bestehen, den in der Regel nur vorübergehenden Widerruf der Abrechnungsgenehmigung ganz oder teilweise zu kompensieren, um nach Ablauf der Wiedererteilungsfrist die Abrechnungsgenehmigung erneut zu beantragen. Dieser Weg dürfte in der Regel kostensparender und u.U. effektiver sein, als die Beschreitung sämtlicher gerichtlicher Instanzen. Dies gilt vor allen Dingen in den Fällen, in denen die Kassenärztliche Vereinigung die sofortige Vollziehung des Widerrufs der Genehmigung angeordnet hat.

\section{René T. Steinhäuser \\ Rechtsanwalt \\ Dr. Peter Wigge \\ Rechtsanwalt \\ Fachanwalt für Medizinrecht}

\author{
Rechtsanwälte Wigge \\ Neuer Wall 44 \\ 20354 Hamburg \\ Tel.: 040/3398705-90 \\ Fax: 040/3398705-99 \\ Internet: www.ra-wigge.de \\ E-Mail: kanzlei@ra-wigge.de
}

